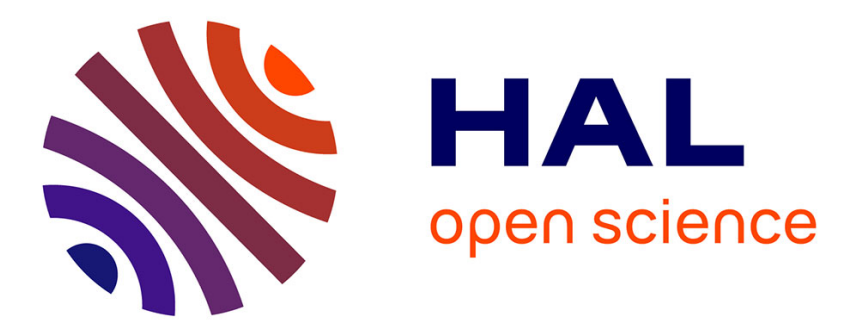

\title{
Les banquiers centraux dans la construction européenne : introduction
}

Frédéric Clavert, Olivier Feiertag

\section{To cite this version:}

Frédéric Clavert, Olivier Feiertag. Les banquiers centraux dans la construction européenne : introduction. Histoire, économie et société, 2011, 30e année (4), pp.3-9. 10.3917/hes.114.0003 . halshs01100460

\section{HAL Id: halshs-01100460 \\ https://shs.hal.science/halshs-01100460}

Submitted on 7 Jan 2015

HAL is a multi-disciplinary open access archive for the deposit and dissemination of scientific research documents, whether they are published or not. The documents may come from teaching and research institutions in France or abroad, or from public or private research centers.
L'archive ouverte pluridisciplinaire HAL, est destinée au dépôt et à la diffusion de documents scientifiques de niveau recherche, publiés ou non, émanant des établissements d'enseignement et de recherche français ou étrangers, des laboratoires publics ou privés. 


\title{
LES BANQUIERS CENTRAUX DANS LA CONSTRUCTION EUROPÉENNE : INTRODUCTION
}

\author{
Frédéric Clavert et Olivier Feiertag
}

Armand Colin | Histoire, économie \& société

\section{1/4 - 30e année \\ pages 3 à 9}

ISSN 0752-5702

Article disponible en ligne à l'adresse:

http://www.cairn.info/revue-histoire-economie-et-societe-2011-4-page-3.htm

Pour citer cet article :

Clavert Frédéric et Feiertag Olivier, «Les banquiers centraux dans la construction européenne : introduction », Histoire, économie \& société, 2011/4 30e année, p. 3-9. DOI : 10.3917/hes.114.0003

Distribution électronique Cairn.info pour Armand Colin.

(c) Armand Colin. Tous droits réservés pour tous pays.

La reproduction ou représentation de cet article, notamment par photocopie, n'est autorisée que dans les limites des conditions générales d'utilisation du site ou, le cas échéant, des conditions générales de la licence souscrite par votre établissement. Toute autre reproduction ou représentation, en tout ou partie, sous quelque forme et de quelque manière que ce soit, est interdite sauf accord préalable et écrit de l'éditeur, en dehors des cas prévus par la législation en vigueur en France. II est précisé que son stockage dans une base de données est également interdit. 


\title{
Les banquiers centraux dans la construction européenne : introduction
}

\author{
par Frédéric Clavert et Olivier Feiertag
}

Les banquiers centraux semblent aujourd'hui solidement installés au cœur de notre monde contemporain. L'unification monétaire européenne à l'orée du XXI ${ }^{\mathrm{e}}$ siècle n'a pas correspondu pour les banques centrales à « une fin de l'histoire ». La création de la Banque centrale européenne (BCE) le $1^{\text {er }}$ juin 1998 et la formation le $1^{\text {er }}$ janvier 1999 du Système européen de banques centrales, en application du Traité de Maastricht, marquent sans aucun doute une étape importante dans le cours d'une évolution multiséculaire, commencée au début du XIX $\mathrm{X}^{\mathrm{e}}$ siècle pour les plus anciennes des banques centrales en Europe, voire plus tôt encore comme dans le cas de la Banque d'Angleterre créée en 1694 ou encore de la Banque de Suède, la doyenne des banques centrales, fondée dès 1668. La longue histoire des banques nationales d'émission, comme on les appelle avant 1914, est inséparable de l'histoire de la construction des États-nation. La Banque de France, comme le rappelle Vincent Auriol en 1936 comme encore Georges Pompidou en 1970, devait être et demeurer « la Banque de la France».

Dans cette optique, la réalisation de la monnaie unique européenne a été d'emblée analysée, pour s'en réjouir ou s'en lamenter, comme une amputation décisive du plus régalien des droits, celui de battre monnaie, mais aussi, le fait a été moins souvent explicité, de la capacité des États à continuer à trouver auprès de leur «banquier naturel », les avances de trésorerie dont ils avaient toujours eu historiquement le plus grand besoin. Le rôle des différentes banques centrales nationales depuis le déclenchement de la première crise financière globale en $2007^{1}$ et, plus encore, de la première crise majeure à laquelle est confronté l'euro depuis 2010, montre que l'hypothèse du dépérissement des banques centrales associé à la perspective de « la fin des territoires », envisagée par Bertrand Badie dès $1995^{2}$, mérite d'être examinée plus avant.

Deux faits importants de l'histoire immédiate de la crise de l'euro doivent d'ores et déjà être soulignés : la défiance des marchés de l'argent envers certains États de l'Euroland se nourrit, on le sait, du niveau de leur dette publique jugé à tort ou à raison « insoutenable ». Tout se passe comme si le ratio de la dette publique rapportée au PIB de chaque État avait

1. Cf. Youssef Cassis, « La crise financière de 2008 : une perspective historique », dans V. Dujardin et al., La Crise économique et financière de 2008-2009, Bruxelles, PIE-Peter Lang, 2010, p. 41-51.

2. Bertrand Badie, La Fin des territoires, Essai sur le désordre international et sur l'utilité sociale du respect, Paris, Fayard, 1995. 
remplacé le taux d'inflation qui prévalait avant le passage à l'euro, comme signal d'alarme aux yeux des marchés. Mais cette défiance se nourrit autant sinon plus de la prise en compte du solde final de la balance des paiements des États. La détérioration continue de la balance des paiements de la Grèce, autant sinon plus que le niveau de sa dette souveraine, a alimenté la défiance des marchés. La publication par la BCE de la balance des paiements de la zone euro n'a donc pas pour autant fait disparaitre la réalité des balances des paiements nationales dont la confection relève d'ailleurs principalement dans tous les pays de la banque centrale nationale. Cette persistance du cadre de la «comptabilité nationale »comme on disait dans les années 1950 - incite à réévaluer l'hypothèse de l'effacement historique du cadre des économies nationales et du dépérissement des banques centrales nationales.

\section{Pour une histoire des banquiers centraux}

D'une importance croissante depuis les années 1980, le rôle joué par les banques centrales dans les évolutions et la régulation des systèmes financiers nationaux comme des relations monétaires internationales n'est pas contestable. Cette importance explique que le sujet des banques centrales et les études d'économie monétaire consacrées au central banking soient à l'origine d'une immense bibliographie qui remplit des bibliothèques entières.

Et pourtant, paradoxalement, si les banques centrales ont été analysées de manière très détaillée, les banquiers centraux demeurent, eux, encore mal connus ${ }^{3}$. Ces hommes - et de plus en plus aujourd'hui ces femmes -, qui travaillent dans les banques centrales et y exercent les différents métiers, eux aussi mal connus, des banquiers centraux, sont pourtant au cœur de l'histoire des banques centrales et leur réalité changeante conditionne largement la validité des multiples modèles engendrés par les théories successives du central banking.

Les banques centrales, comme le soulignait Alan Greenspan, au moment où il a été nommé à la tête de la Fed, le système de banques centrales des États-Unis, demeurent, pour tous les observateurs qui sont à l'extérieur du monde des banquiers centraux, a black box $x^{4}$, des boîtes noires. Il s'en faut cependant, et de loin, qu'elles soient des boîtes vides. Les banques centrales recouvrent historiquement quatre dimensions qu'il importe de bien avoir présentes à l'esprit quand on fait l'histoire des banques centrales.

Tout d'abord, il existe une dimension humaine : il n'y a pas de central banking sans banquiers centraux dont les trajectoires individuelles et collectives ont varié avec le temps 5 . L'étude des carrières de ces hommes et de ces femmes ne met-elle pas parfaitement en lumière la question de la position des banques centrales entre les pouvoirs politiques et les compétences techniques des marchés ? Sous cet angle, c'est aussi, au-delà des personnels, la réalité des banques centrales comme organisation qu'il faut analyser, à

3. La publication de l'ouvrage de John Singleton a très récemment ouvert une brèche significative dans cette relative méconnaissance en mettant clairement en évidence la réalité professionnelle des banquiers centraux dans le monde : Central Banking in the Twentieth Century, Cambridge, Cambridge University Press, 2011.

4. Alan Greenspan, The Age of Turbulence : Adventures in a New World, New York, The Penguin Press, 2007, p. 99.

5. Frédéric Lebaron a travaillé sur une prosopographie des banquiers centraux dont les premiers résultats ont été exposés au colloque European Power Elite : Frédéric Lebaron, «Quelques résultats et réflexions sur la prosopographie des banquiers centraux », colloque European Power Elite : où en est la sociologie politique de l'Europe ?, Paris 1, Centre Malher, 10 juin, discutante : M. de Lassalle. À notre connaissance, il n'y a pas eu de publication de cette intervention, mais l'on pourra toutefois consulter Frédéric Lebaron, « Central bankers in the contemporary global field of power : a "social space" approach », The Sociological Review, avril 2008, vol. 56, p. 121-144. 
travers les mutations de leurs structures, donc de leurs métiers, comme des modalités de leur gouvernance interne.

La seconde dimension est économique. Parce que l'objet principal d'une banque centrale est d'émettre et de faire circuler sous son contrôle la monnaie, l'action des banques centrales est un élément crucial des évolutions non seulement des systèmes financiers dont elles sont la clef de voûte comme prêteur en dernier ressort du système, mais aussi, au-delà, des évolutions de tous les marchés : marchés des capitaux, mais aussi des biens et même du travail.

En troisième lieu, les banques centrales ont une dimension juridique. Elles sont avant tout des entités légales uniques dont l'identité juridique, elle aussi changeante avec le temps, règle directement leur capacité d'intervention, leurs outils mais aussi la question majeure de leur indépendance.

Enfin, la dimension culturelle ne doit pas être oubliée. Quelle est la culture commune des banquiers centraux, si toutefois elle existe ? Il faut ici poser la question de leurs bagages théorique et pratique, de leurs représentations (mots, images, concepts) liées à l'histoire et à la mémoire des banquiers centraux. De cette façon, l'on pourra espérer restituer l'évolution de leur outillage mental, pour reprendre la belle expression de Lucien Febvre, en amont de toute politique monétaire.

La prise en compte des multiples facettes des banques centrales fournit une grille d'analyse particulièrement fertile pour le grand chantier de l'histoire du rôle des banquiers centraux dans la construction européenne.

\section{Banques centrales et construction européenne}

Elle permet d'abord d'explorer le problème très disputé de la part respective qui revient aux logiques politiques et aux dynamiques techniques - supposées a-politiques - dans les étapes de la construction européenne, singulièrement dans l'histoire des relations monétaires européennes, y compris bien entendu dans ce grand fait, dont l'histoire est loin d'avoir été encore écrite, qu'est l'unification monétaire européenne. Où sont les banquiers centraux entre le technicien et le politique, qu'on désigne parfois de manière péjorative comme le politicien ? Le pouvoir des banquiers centraux existe-t-il, au-delà du mythe du « complot » des banquiers internationaux, si vite endossé à chaque crise financière par les opinions et les médias en mal de bouc émissaire?

L'histoire des banquiers centraux dans la construction européenne, en second lieu, permet d'aborder de manière tout à fait cruciale la problématique qui s'impose aujourd'hui à n'en pas douter dans le champ des sciences humaines, celle de la mondialisation. Cette notion, pour peu qu'on la définisse un peu rigoureusement et qu'on ne s'en tienne pas à une approche seulement quantitative qui aboutit à distinguer des mondialisations à toutes les époques de l'histoire de l'humanité, met en effet en pleine lumière la question peutêtre principale du rôle qui a été celui des banquiers centraux dans l'histoire de l'Europe monétaire : quelle part ont pris les banques centrales dans le processus qui au long du $\mathrm{XX}^{\mathrm{e}}$ siècle, mais singulièrement depuis les années 1960-1970, semble bien avoir opposé les protections des États-nations, largement fondée en dernière analyse sur la souveraineté monétaire, aux dynamiques du marché mondial ?

Enfin, s'intéresser aux banquiers centraux d'un point de vue historique, c'est-à-dire quasi anthropologique, c'est traiter d'un autre problème clef de l'histoire de la « construction européenne », de ses échecs comme de ses réussites : quelle est l'histoire de la relation entretenue par les banquiers centraux vis-à-vis de leur intérêt national respectif ? Les banques centrales ont commencé par être des banques d'émission nationales - c'est encore 
comme cela qu'on les désigne en avril 1922 à la conférence de Gênes et c'est d'ailleurs à cette période, au lendemain de la Première Guerre mondiale, que sous la forte influence du gouverneur de la Banque d'Angleterre, Montagu Norman, on commence à les percevoir plus collectivement à travers l'image du « petit peuple des banques centrales ${ }^{6}$ ».

Leur transformation progressive en banques centrales, y compris à travers le phénomène de leurs duplications durant tout l'entre-deux-guerres dans la plupart des États-nation qui en étaient encore dépourvus, a-t-il abouti à faire des banquiers centraux une communauté épistémique $^{7}$, de nature transnationale, selon un concept cher aux politologues et dont la réalité dans les milieux de la SDN comme sans doute des institutions européennes semble bien pouvoir être attestée ${ }^{8}$ ?

Dans un numéro de la revue International Organization de 1992, Haas et Adler définissent la communauté épistémique comme « un réseau de professionnels de disciplines et d'horizons divers, qui (1) partagent un ensemble de convictions normatives et de principes donnant des raisons de l'ordre des valeurs à l'action sociale des membres de la communauté ; (2) partagent des convictions causales, tirées de leur analyse de pratiques conduisant ou contribuant à un ensemble central de problèmes dans leur domaine et qui servent alors de base pour élucider les multiples liens entre les actions politiques possibles et les résultats souhaités ; (3) partagent des notions de validité - c'est-à-dire des critères intersubjectifs définis en interne pour évaluer et valider les connaissances dans leur domaine d'expertise ; (4) ont une entreprise politique commune - c'est-à-dire un ensemble de pratiques communes associé à un ensemble de problèmes vers lesquels leur compétence professionnelle est orientée, probablement avec la conviction que cela améliorera le bien-être de l'humanité. »9

\section{Premiers résultats de la recherche}

Si nous ne prétendons pas compléter ou détailler cette définition, nous pensons que ce numéro permettra de répondre à une partie des problématiques posées par cette définition de la communauté épistémique appliquée aux banquiers centraux, tout en donnant une vision plus historique de cette communauté. Cette notion pose certaines questions auxquelles les articles qui suivent pourront pour partie répondre.

La définition de Haas parle d'un réseau de professionnels, ce qui nous pousse à nous interroger sur plusieurs points. Le premier est la question de la définition du banquier central. Prenons l'exemple d'Alexandre Lamfalussy : banquier privé, passé à la Banque des Règlements internationaux dont il devient directeur général il est très largement intégré au monde des banquiers centraux comme le montre l'article d'Ivo Maes, et ce bien avant de devenir banquier central lui-même en tant que président de l'institut monétaire européen à partir de 1994. D'autres cas, comme Robert Triffin, économiste, longtemps peu apprécié des banquiers centraux, mais qui marque largement les questions monétaires européennes

6. Philip L. Cottrell, « Norman, Strakosch and the development of central banking : from conception to practice, 1919-1924 », in Philip L. Cottrell (éd.), Rebuilding the Financial System in Central and Eastern Europe, 1918-1994, Ashgate, Scolar Press, 1997.

7. Sur la notion, maintenant bien établie dans le champ des sciences humaines, de communauté épistémique, voir Haas, P. (1992) « Introduction : epistemic communities and international policy co-ordination », International Organization, 46 (1) 1-35 ; Adler E. et Haas P. M. (1992) « Conclusion : epistemic communities, world order, and the creation of a reflective research program », International Organization, 46 (1) 367-90.

8. Amy Verdun, « The role of the Delors Committee in the creation of EMU : an epistemic community ? », Journal of European Public Policy, 2001, vol. 6, n 2, p. 308-328.

9. Peter M. Haas, «Introduction : epistemic communities and international policy co-ordination », op. cit., p. 3. 
dès la fin des années 1950, sont bien moins évidents. Une définition simple des banquiers centraux n'est ainsi pas envisageable.

De plus, l'expression «banquiers centraux » incite implicitement à présupposer un groupe très homogène. Or, il existe des tensions internes, parfois pour des questions nationales, au sein de ce groupe. Si nous prenons l'exemple de l'entre-deux-guerres, la Banque d'Angleterre et son gouverneur, Montagu Norman, et la Reichsbank et son président Hjalmar Schacht, se retrouvent souvent dans un groupe opposé à la Banque de France et la Banque nationale de Belgique, comme le rappelle notamment Frédéric Clavert. S'il y a des valeurs communes et ce, dès les années 1920 au moins, il existe également des lignes de partage.

L'élaboration de ces valeurs communes - en premier lieu l'indépendance des banques centrales, thème qu'une très grande partie des articles de ce numéro aborde et, en premier lieu, celui d'Ileana Racianu - dépend aussi de lieux de sociabilité des banquiers centraux. Comme on peut le remarquer dans les trajectoires de Robert Raymond, Hanspeter Scheller mais aussi, d'une certaine manière, dans celle de Pierre Werner retracée ici par Elena Danescu, certains lieux sont déterminants.

En premier lieu, dès sa fondation en 1930, la Banque des Règlements internationaux, évoquée par Frédéric Clavert, Vincent Duchaussoy, Ivo Maes, Robert Raymond et Hanspeter Scheller, est une institution liant relations formelles entre les banques centrales et leurs gouverneurs, et relations informelles entre les banquiers centraux, notamment à l'occasion des fameux dîners communs qui ont lieu la veille des réunions mensuelles du conseil d'administration de cette banque dont les actionnaires sont les banques centrales.

Mais la BRI n'est pas la seule «institution » internationale qui voit les banquiers centraux se réunir et se concerter - parfois exposer leurs différences. Bien sûr, le Comité des gouverneurs des États membres de la Communauté économique européenne en est un, mais il siège à Bâle et travaille avec la BRI. Le Fonds monétaire international - comme le montrent les articles de Robert Raymond et Hanspeter Scheller -, l'est également. L'impact de la crise actuelle en Grèce sur la zone euro et l'intervention du FMI et de l'Union européenne dans ce pays, montrent que les questions monétaires ont à la fois des logiques nationales, européennes et mondiales. Les banquiers centraux doivent jouer avec ces trois échelles.

De plus, certaines banques centrales exercent plus d'influence que d'autres. La Banque d'Angleterre dans l'entre-deux-guerres - en raison notamment de la personnalité de son gouverneur Montagu Norman - la Bundesbank après-guerre, qui profite d'une part de son statut d'indépendance, d'autre part de la vitalité de l'économie allemande dans la seconde moitié du $\mathrm{XX}^{\mathrm{e}}$ siècle, ont largement dominé les débats sur l'organisation monétaire du continent européen, à deux périodes différentes. Les articles de Vincent Duchaussoy et d'Emmanuel Mourlon-Druol montrent que la Bundesbank, profitant de son statut, a su dominer le Serpent puis le Système monétaire européen, ce qui explique sa forte influence sur l'Union économique et monétaire telle qu'elle a été mise en œuvre dans les années 1990.

La notion d'indépendance de la banque centrale vis-à-vis de l'État, qui est la valeur fondamentale partagée par les banquiers centraux depuis les années 1920 au moins, ne s'est pas imposée facilement aux gouvernements. Ces derniers, même lorsque cette indépendance a été accordée, tente de revenir dessus, au nom des intérêts de l'État, comme le montre l'exemple du gouverneur Burilianu en Roumanie mis en exergue par Ileana Racianu. Cette question déterminante de l'indépendance et des relations des banques centrales à l'État pose aussi la question de la défense des intérêts nationaux par les 
premières dans les hauts lieux de cette communauté des banquiers centraux. Ainsi Hjalmar Schacht défend-il la Nuit des longs couteaux en juillet 1934 à la BRI alors que l'événement, conjugué au règlement de la question des transferts, l'a profondément abattu ${ }^{10}$. Les articles d'Emmanuel Mourlon-Druol et Vincent Duchaussoy démontrent bien le paradoxe de ces institutions financières qui se doivent de défendre la position de leur pays tout en revendiquant une certaine orthodoxie financière de nature proprement transnationale et leur indépendance vis-à-vis de leur gouvernement.

La notion de « communauté épistémique » nous pousse également à nous interroger sur la chronologie et sur l'environnement historique qui a permis l'émergence d'une communauté épistémique des banquiers centraux au fil $\mathrm{du} \mathrm{XX}^{\mathrm{e}}$ siècle.

Le premier temps fort qui nous semble émerger est la Première Guerre mondiale, car les turbulences monétaires fortes des années d'entre-deux-guerres suscitent l'émergence d'un mythe, celui de l'étalon-or. L'histoire de l'organisation monétaire du continent européen au cours du $\mathrm{XX}^{\mathrm{e}}$ siècle ressemble fort à une tentative - inachevée ? - de retour vers la stabilité monétaire perdue et largement idéalisé de l'étalon-or de la fin du XIX ${ }^{\mathrm{e}}$ siècle. Mais la Première Guerre mondiale et ses suites marquent aussi par un endettement international, lié à la guerre, dont le niveau n'a jamais été atteint auparavant. Dettes interalliées ou Réparations politiques, l'année 1919 ouvre une ère monétaire nouvelle. Une première réponse, imparfaite, est finalement trouvée : la création de la Banque des règlements internationaux et l'évolution progressive vers un étalon de change-or, plus politique.

Le deuxième moment est lié au second conflit mondial qui débouche, notamment, sur un nouvel ordre monétaire élaboré à la conférence de Bretton Woods de 1944, qui instaure le Fonds monétaire international, la Banque internationale pour la reconstruction et le développement (l'actuelle Banque mondiale) et l'étalon de change-or. Mais ce dernier est dans un premier temps un étalon dollar. Les États européens, incapables d'assurer la convertibilité or de leurs monnaies, s'organisent par la création de l'Union européenne des paiements (UEP), qui fait suite au plan Marshall. L'UEP est un moment fort de la coopération monétaire européenne, vue, après sa disparition en 1958, comme un exemple réussi de coopération monétaire par Jean Monnet ou Robert Triffin ${ }^{11}$.

La période suivante s'ouvre avec l'entrée en vigueur de l'Accord monétaire européen, qui remplace l'UEP, à partir du $1^{\mathrm{er}}$ janvier 1958. Les années qui suivent sont, d'une certaine manière, une époque d'application réelle de l'étalon de change-or en Europe, avec certaines limites à la convertibilité des monnaies. Si le système monétaire international de Bretton Woods se révèle satisfaisant, il est néanmoins très asymétrique en faveur du dollar, ce qui ouvre une première série de discussions au sein de la Communauté économique européenne, qui débouchent notamment sur le chapitre VIII du mémorandum de la Commission de l'automne 1962, très influencé par le Commissaire français Robert Marjolin ${ }^{12}$, chargé de l'économie et des finances et vice-président de la Commission européenne de la CEE. Ce rapport donne naissance au Comité des gouverneurs des banques centrales des États membres de la CEE en 1964, qui siège à Bâle et qui est largement évoqué dans la plus

10. Frédéric Clavert, Hjalmar Schacht, financier et diplomate : 1930-1950, Bruxelles, PIE - Peter Lang, 2009, p. 231.

11. Voir Gérard Bossuat, «Questions sur l’identité monétaire européenne à travers les positions de Jean Monnet, Robert Triffin et Pierre Mendès France », in Éric Bussière et Michel Dumoulin, Milieux économiques et intégration européenne au XX siècle, Arras, Artois Presses Université, p. 105-113.

12. Commission européenne, Mémorandum de la Commission sur le programme d'action de la Communauté pendant la deuxième étape. Bruxelles : Service des publications des Communautés européennes, 1962, chapitre VIII - Politique monétaire. 
grande partie des articles de ce numéro. Toutefois, dans les années 1960, les banquiers centraux restent principalement convaincus que les questions monétaires se gèrent au niveau mondial plutôt qu'au niveau européen.

Mais à la fin des années 1960, le SMI rentre dans une zone de turbulences, qui mènent à la fin de la convertibilité en or du dollar en 1971 et à la disparition de l'étalon de change-or par les accords de la Jamaïque de 1976. L'échelon européen devient alors séduisant pour le traitement des affaires monétaires. En décembre 1969, le sommet de La Haye convoque le Comité Werner - dont l'article d'Elena Danescu, reposant sur des archives inédites, donne un éclairage nouveau - qui débouche sur la création d'un Serpent monétaire moins ambitieux que les conclusions du Comité et qui fonctionne mal. Emmanuel MourlonDruol montre très bien que malgré ces dysfonctionnements du Serpent, avec les sorties du Royaume Uni, de l'Italie, de la France, les réflexions sur l'intégration monétaire continuent et aboutissent en 1979 sur la création du Système monétaire européen (SME).

La phase qui suit voit le SME fonctionner raisonnablement bien. Mais la volonté d'intégration monétaire - notamment dans le cadre du couple franco-allemand - s'accroît. Le SME reste un système imparfait et les multiples dévaluations du franc français, notamment, encouragent les Européens à passer à l'intégration monétaire. L'Acte unique, signé en 1986 et entré en vigueur le $1^{\text {er }}$ juillet 1987 fait référence à l'Union économique et monétaire. Il ouvre ainsi la voie au comité Delors et, ensuite, à la signature, en février 1992, du traité de Maastricht qui enclenche le processus de l'Union économique et monétaire.

Deux des moments de cette histoire longue nous semblent plus importants que les autres. 1919 car l'endettement international atteint un niveau inédit, difficilement « soutenable » sans coopération entre États mais également entre banques centrales, mais aussi le début des années 1990, lorsque le volume des transactions sur un marché de l'argent devenu «global» dépasse la masse de manœuvre des banques centrales nationales. C'est cet effet de seuil qui est à l'origine de la très grave crise monétaire qui frappe l'Europe en 1992-1993.

Ces premiers résultats n'épuisent pas, et de loin, un sujet que l'ouverture progressive des archives publiques et privées, la multiplication des témoignages oraux ne cesse d'enrichir. Il ne fait guère de doute qu'ainsi comprise, l'histoire des banquiers centraux figure au premier plan d'une histoire du temps présent dont la nécessité est confirmée par les profondes mutations dans lesquelles sont sans conteste plongées depuis une trentaine d'années les sociétés européennes. C'est à ce vaste chantier que les différentes contributions, d'historiens et d'acteurs, rassemblées ici voudraient, pour leur part, contribuer. 